\title{
Polystyrene Beads-Based System for Optical Sensing using Spiropyran Photoswitches
}

\author{
Silvia Scarmagnani ${ }^{a}$, Zarah Walsh $^{b}$, Conor Slater $^{a}$, Nameer Alhashimy ${ }^{a}$, Brett Paull ${ }^{b}$, Mirek Macka $^{b}$, \\ Dermot Diamond $^{a *}$
}

\author{
${ }_{5}$ Received (in XXX, XXX) 1st January 2007, Accepted 1st January 2007 \\ First published on the web 1st January 2007 \\ DOI: $10.1039 / b 000000 x$
}

Spiropyran derivatives have been immobilised on the surface of polystyrene microbeads using different immobilisation strategies. These functionalised polymeric beads can be reversibly switched between the colourless 10 inactive spiropyran (SP) and highly coloured (purple) active merocyanine (MC) forms using low power light sources, such as light emitting diodes (LEDs). A UV-LED $(375 \mathrm{~nm})$ is used for the SP to MC conversion, and a white LED (430-760 nm) for the reverse MC to SP conversion. The photochromic behaviour of the coated beads has been characterised using different LEDs and reflection spectroscopy, employing optic fibres and an in-house designed holder. Investigations into the metal-ion binding behaviour of the spiropyran modified microbeads have shown that ${ }_{15} \mathrm{Cu}^{2+}$ ions cause an appreciable colour and spectral change when brought into contact with the beads in the MC form, suggesting that a significant interaction is occurring. However, the $\mathrm{Cu}^{2+}$ ions can be completely expelled by photonicconversion of the beads into the inactive SP form using a white LED. This sequence has been successfully repeated six times suggesting that it is possible to cycle through activation of the functionalised beads from a non-binding to a binding form (SP to MC) using a UV-LED, allow binding with $\mathrm{Cu}^{2+}$ ions to occur, and subsequently, expel the bound 20 ions and regenerate the passive SP surface using a white-LED. Other metals, such as calcium, do not cause any appreciable colour or spectral change over the same concentration range and in the presence of the same anion (final concentration $7.1 \times 10^{-4} \mathrm{M}$ nitrate salt in ethanol). The system is therefore self-indicating in terms of whether the active MC or inactive SP forms are present, and whether $\mathrm{Cu}^{2+}$ ions are bound to the $\mathrm{MC}$ form. In principle, therefore, these functionalised beads could form the basis of a photoswitchable stationary phase for metal ion binding and

25 detection: irradiation of the stationary phase with UV-LEDs causes retention of guest species due to the presence of the MC form, while subsequent exposure to white LEDs causes release of guest species into the mobile phase.

Keywords: photoswitchable surfaces, adaptive materials, functionalised beads, ion-binding, spiropyran.

30

\section{Introduction}

In science, control of interactions at the molecular scale is the ultimate goal, as this in turn determines all macro scale behaviour. For example, switchable or adaptive surfaces can be 35 externally controlled at a molecular level by changing the state of switchable molecule between an active (binding) or passive (nonbinding) state, enabling or inhibiting its capability to bind target molecules. Organic photochromic compounds are particularly interesting in this regard, as they offer a potential route to new 40 multifunctional materials that take advantage of photo-reversible interconversion between two thermodynamically stable states with different polarities and geometries ${ }^{1}$. Among these, spiropyran derivatives are a useful starting point, as they have been very well studied for many years, and exhibit a striking 45 colour change when switched between the spiropyran (SP) and merocyanine forms (MC); i.e. they are inherently self-indicating. The colourless, uncharged, passive (i.e. non ion-binding) SP form exhibits an absorption spectrum with an actinic band in the UV range 320-380 $\mathrm{nm}$. Exposure to UV radiation in this range leads 50 to the formation of the strongly coloured, zwitterionic, active merocyanine form (MC), in which the extended conjugation leads to a dramatic shift in the UV-absorption, with a maximum wavelength around $560 \mathrm{~nm}$ (depending on the polarity of the immediate environment $)^{2}$. Upon irradiation, the C-O spiro bond 55 in the SP form is cleaved heterolytically and the "spiro" carbon adopts the planar MC configuration. When the UV source is removed, the molecules gradually relax to their ground $\operatorname{state}^{3}$, the carbon-oxygen bond renews and the molecule returns to the colourless SP form. The MC form can be regarded as a resonance ${ }_{60}$ hybrid between a zwitterionic form with localised charges and an apolar polyenic form or quinonoid. The zwitterionic form contributes significantly to the structure of the open form because the aromaticity of the oxygen-bearing ring is lost in the neutral form $^{2}$ (Fig. 1). From this discussion, it is clear that molecular${ }_{65}$ Switches based on spiropyran-like molecules, open the possibility of generating surfaces whose physico-chemical properties can be controlled under an external photonic stimulation.

It has been demonstrated that low-power light sources such as UV and green light emitting diodes (LEDs) can switch 70 spiropyran-modified surfaces between the SP and MC forms ${ }^{4}$. Furthermore, the MC form possesses a phenolate group which is a rather weak binding site for certain cations, such as cobalt ${ }^{4}$. Upon binding, the colour changes again, and afterwards the guest can be expelled using a green LED to reform the original inactive ${ }_{75} \mathrm{SP}$. The binding of many metals with spiropyran and various spiropyran derivatives has been abundantly evaluated, either when the photochromic dye is in solution ${ }^{5,6}$ or within a polymeric matrix 478910 . Using spiropyran moieties therefore it should be possible to generate 'adaptive' or switchable surfaces 80 that bind guest species only when irradiated with UV-light ${ }^{4}$.

Bead-based systems are attractive for many applications, as they are essentially bi-phase systems that combine many of the advantages of solids and liquids, such as providing surfaces that can be readily functionalised using well-established chemistries, 85 while also being highly mobile in suspension like a fluid, yet easily separated from a true liquid-phase. 


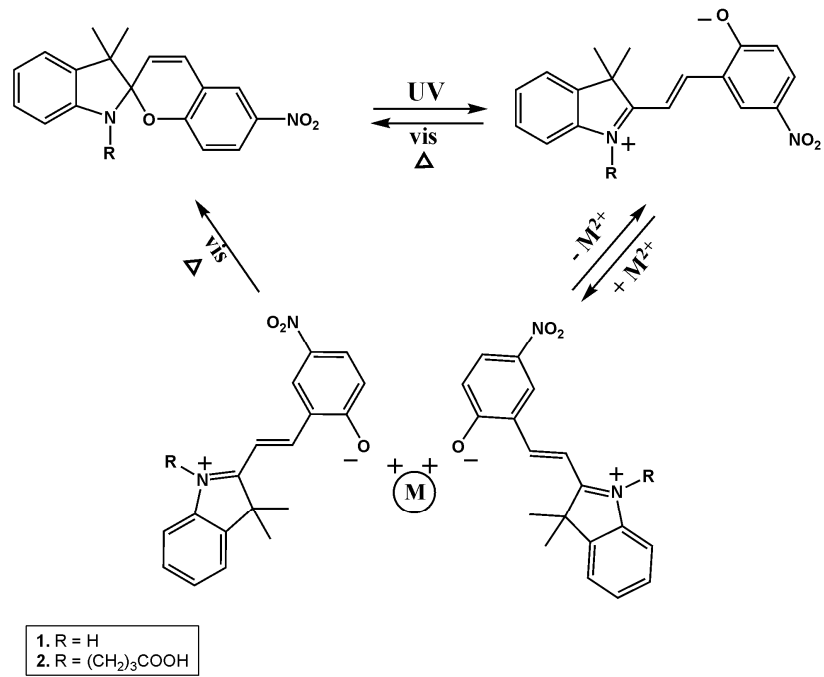

Figure 1: Switching equilibrium between the SP, MC and MC-metal 5 complex.

Micro- and nano-beads provide a higher surface area than flat surfaces for chemical reactions and hence kinetics are enhanced 10 compared to conventional surfaces ${ }^{11}$.

The low density of the typical polymer matrices used allows binding kinetics which can be compared to those of solution based systems and their large surface area and greater density permit rapid and highly efficient binding of target species.

15 Although it has been demonstrated that polystyrene microbeads are individually transparent and colourless, their suspension has a strong opaque milky-white colour in natural light as they are very effective light scatterers ${ }^{12}$. Polystyrene microbeads with diameter greater than $1 \mu \mathrm{m}$ tend to be relatively easy to separate from the 20 suspending solution by centrifugation which greatly simplifies purification stages during multi-step synthetic procedures. In this particular study, although the SP functionalised $2 \mu \mathrm{m}$ diameter microbeads appeared coloured when in the MC form, the high degree of light scattering inhibits transmittance measurements.

${ }_{25}$ Surface reflection spectroscopy is an alternative powerful instrument to study dyes and colours and it has been used for a wide range of applications such as determining chromic behaviour of thermochromic organic compounds in crystalline powders ${ }^{13}$, measuring physically adsorbed colorimetric reagents 30 on polystyrene beads for solid phase extractions ${ }^{14}$, developing fibre optic sensors based on the immobilisation of a coloured chelating agent on a cellulose membrane ${ }^{15}$, reflection studies on the surface of spiropyran monolayers for detection of the formation of aggregates from the merocyanine form ${ }^{16}$ and 35 pigment detection on biological samples ${ }^{17} 18192021$.

These functionalised beads therefore should exhibit switchable binding of certain ions, which could have interesting potential for photo-controlled retention and release of bound species. Furthermore, this behaviour can also be easily location and time 40 specific, depending on where and when the microbeads are irradiated.

\section{Experimental}

Materials and instruments

50 Polybead carboxylate microbeads $2.035 \mu \mathrm{m}$ diameter, $2.79 \%$ solid contents, were purchased from PolySciences Inc., N-(3dimethylaminopropyl)-N'-ethylcarbodiimide hydrochloride (EDC hydrochloride), 2-(N-morpholino)ethanesulfonic acid hydrate (MES hydrate), 1,8-diaminooctane, 1,4-diaminobutane, calcium 55 nitrate hydrate, copper(II) nitrate trihydrate, 1',3'-dihydro1',3',3'-trimethyl-6-nitrospiro benzopyran-2, 2'-(2H)-indole (1, Fig. 1) were purchased from Sigma Aldrich (Ireland) and 1'-(3carboxypropyl)-3',3'-dimethyl-6-nitrospiro(2H-1)benzopyran-

2,2'-(2H)-indole (2, Fig. 1) was synthesised as described 60 elsewhere ${ }^{22}$.

Homogeneous suspensions of microbeads were generated using a Bransonic Ultrasonic Cleaner 5510 from Branson Ultrasonics Corporation, USA. Sample spinning was carried out using a ROTOFIX 32 centrifuge (Global Medical Instrumentation, USA.) ${ }_{65} \mathrm{UV}(375 \mathrm{~nm})$, white $(430-760 \mathrm{~nm})$, blue $(430 \mathrm{~nm})$, green $(525$ $\mathrm{nm})$, red $(630 \mathrm{~nm})$ LEDs were purchased from Roithner Laser Technik, Austria. The UV light source used for the solution studies was a BONDwand UV-365nm obtained from Electrolyte Corporation, USA.

70 Absorbance spectra were recorded using a Well Plate Spectrometer (Medical Supply Co., Ireland).

Reflectance spectra were recorded using a miniature diode array spectrophotometer $\left(\mathrm{S} 2000^{\circledR}\right)$ combined with an FCR-7UV200-2 reflection probe ( 7 X 200 micron cores) and a DH-2000-FSH 75 deuterium halogen light source (215-1700 nm, Ocean Optics Inc., Eerbeek, Netherlands). A white reflectance standard WS-1-SL was used to standardise the measurements at $100 \%$ reflectance ${ }^{21}$ 23 (Ocean Optics Inc., Eerbeek, Netherlands).

An in-house designed vial holder was fabricated using a 3D 80 printer (Stratasys, USA) in black acrylonitrile butadiene styrene co-polymer (ABS) plastic in order to minimise interferences from ambient light. The two parts of the holder (lid and the main body) were designed using ProEngineer CAD/CAM software package.

${ }_{85}$ Spectroscopic studies on response characteristics of $\mathrm{MC}-\mathrm{Cu}^{2+}$ and $\mathrm{MC}-\mathrm{Ca}^{2+}$ complexes formation in ethanol solutions

Solution studies were performed using ethanolic solutions of spiropyran and the nitrate salts of copper and calcium. Stock solutions $\left(10^{-3} \mathrm{M}\right)$ of spiropyran $(\mathbf{1})$ in ethanol were prepared and 90 stored in the dark at room temperature. Stock solutions of $10^{-2}$, $10^{-3}, 10^{-4} \mathrm{M}$ calcium and copper nitrate were prepared by dissolving the corresponding nitrate salt in ethanol.

Absorbance spectra were recorded using a plate well reader with the following procedure:

95 1. Aliquots of $1.5 \mathrm{ml}$ of spiropyran stock solution were placed in plate wells.

2. The solutions were irradiated for 1 minute with UV light and the spectra were recorded immediately after.

3. In all the plate wells, except for one, $0.05 \mathrm{ml}$ of each metal stock solution at differing concentrations was added at the same time using a multiple channel automatic pipette. Final concentrations: $3.2 \times 10^{-6} \mathrm{M}$ (from $0.05 \mathrm{ml}$ of $10^{-4} \mathrm{M}$ metal stock solution), $3.2 \times 10^{-5} \mathrm{M}$ (from 0.05 $\mathrm{ml}$ of $10^{-3} \mathrm{M}$ stock solution), $3.2 \times 10^{-4} \mathrm{M}$ (from $0.05 \mathrm{ml}$ of $10^{-2} \mathrm{M}$ stock solution). In the last well $0.05 \mathrm{ml}$ of ethanol was added and the sample kept as a reference.

4. The absorbance spectra of the solutions were taken immediately after the addition. 


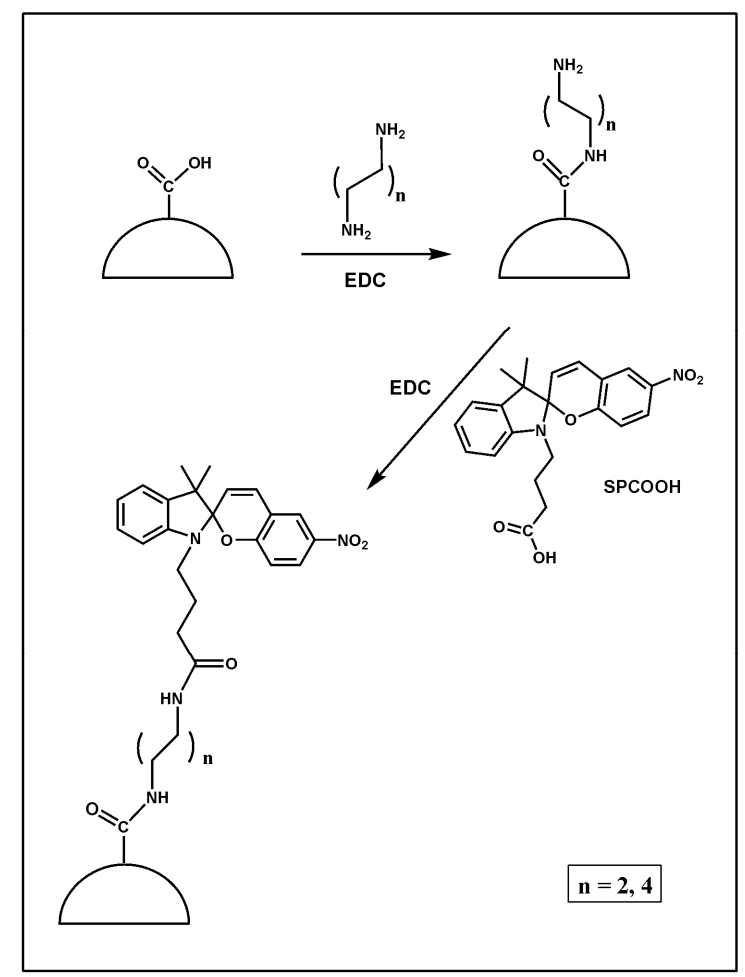

Figure 2: Scheme for the covalent immobilisation of spiropyran on the surface of the polystyrene microbeads.

5

Covalent immobilisation of spiropyran on the surface of polystyrene microbeads using an eight and four carbon spacer

10 A sample of a $0.5 \mathrm{ml}$ suspension of polystyrene carboxylated microbeads $(0.01 \mathrm{~g})$ in water was diluted with $3 \mathrm{ml}$ of a $1 \mathrm{M}$ solution of MES in deionised water and stirred at room temperature for 5 minutes. $1 \mathrm{ml}$ of a $45 \mathrm{mg} \mathrm{ml}^{-1}$ aqueous solution of EDC was then added and the mixture stirred for 1 hour at room 15 temperature.

After $1 \mathrm{ml}$ of $35 \mathrm{mg} \mathrm{ml} \mathrm{m}^{-1}$ of aqueous solution of 1,8diaminooctane (or $1 \mathrm{ml}$ of a $20 \mathrm{mg} \mathrm{ml}^{-1}$ aqueous solution of 1,4-

diaminobutane) was added and the reaction mixture was stirred for 24 hours at room temperature. The microbeads were then 20 washed 3 times with water and 6 times with ethanol.

The washing procedure consists of a four step process:

1. Centrifugation of the suspension for 3 minutes at 4000 $\mathrm{rpm}$.

2. Removal of the supernatant, addition of $4 \mathrm{ml}$ of fresh solvent.

3. Sonication of the suspension for 5 minutes.

4. Subsequent further centrifugation.

Separately, $2 \mathrm{ml}$ of an $18 \mathrm{mg} \mathrm{ml}^{-1}$ solution of 2 in ethanol was 30 prepared and added to $1 \mathrm{ml}$ of $15 \mathrm{mg} \mathrm{ml}^{-1}$ solution of EDC in ethanol. The reaction mixture was stirred for half an hour at room temperature in the dark. A $1 \mathrm{ml}$ suspension of the microbeads in ethanol which were previously functionalised with the amino linker was added to the spiropyran/EDC solution and the reaction 35 mixture was stirred for 72 hours at room temperature in the dark (Fig. 2). Finally, the spiropyran functionalised microbeads were profusely washed 10 times with ethanol following the above reported procedure and stored at $4{ }^{\circ} \mathrm{C}$ in the dark.

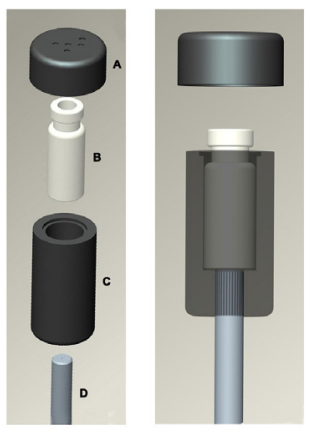

Figure 3: In-house designed vial holder with a recess at the bottom for inserting the probe. The lid blocks any ambient light from reaching the probe. Both parts were printed in black ABS. A) Lid; B) Glass vial containing the sedimented layer of polystyrene microbeads; C) Main 45 body; D) Reflectance probe.

Physical adsorption of spiropyran on the surface of polystyrene microbeads

${ }_{50}$ In order to compare the characteristics of covalently functionalised beads, a sample of polystyrene beads with physically adsorbed $\mathbf{2}$ was prepared.

An aliquot of a $0.5 \mathrm{ml}$ suspension of polystyrene carboxylated microbeads $(0.01 \mathrm{~g})$ in water was diluted with $3 \mathrm{ml}$ of ethanol and

55 added to $2 \mathrm{ml}$ of an $18 \mathrm{mg} \mathrm{ml}^{-1}$ ethanolic solution of $\mathbf{2}$ and the mixture was stirred for 72 hours in the dark at room temperature. The beads were then washed 10 times with ethanol following the above reported procedure and stored at $4{ }^{\circ} \mathrm{C}$ in the dark.

\section{Optical properties of the spiropyran coated microbeads}

\section{Reflectance measurements}

${ }_{65}$ Measurements were performed using optically transparent glass vials and a specially designed vial and fibre optic probe holder (Fig. 3).

The sample was prepared for analysis by centrifugation of a 1.3 $\mathrm{ml}$ suspension containing $0.01 \mathrm{~g}$ of polystyrene microbeads in 70 ethanol for 5 minutes at $4000 \mathrm{rpm}$ to form a homogeneous thin layer of microbeads in the bottom of a glass vial. The vial holder consists of a hollow cylinder in which the vial can be inserted, with a recess at the bottom for inserting the reflectance probe. The unit is completed with a lid that snaps over the holder which 75 has a recess on the underside for securing a vial by its cap. This design allows the vial to be easily inserted and removed, and is very effective at blocking any ambient stray light. Both parts were printed in black ABS plastic.

The vial is placed into the holder, while the reflectance probe is 80 positioned perpendicularly to the microsphere layer. For reflectance measurements, the halogen lamp is used (360-1700 $\mathrm{nm})$. Illumination with the deuterium lamp (215-400 $\mathrm{nm})$ should be avoided as the SP-functionalised beads are very UV sensitive and will switch to the MC form, and therefore affecting the ${ }_{85} \mathrm{SP} \leftrightarrow \mathrm{MC}$ equilibrium during measurements. In contrast, recording spectra of the $\mathrm{MC}$ form over a few seconds using the tungsten lamp doesn't affect the equilibrium as strongly, and the spectra can be easily detected without a rapid reconversion to the SP form. Finally, since the MC peak appears around $560 \mathrm{~nm}$ and 90 this wavelength falls in the visible region, the spectra can simply be detected using just this part of the spectrum. 
The recorded spectra were processed by the Ocean Optics software against a previously recorded reference spectrum of a totally reflective Ocean Optics standard in the dark at the same distance from the reflectance probe 2124 .

${ }_{5}$ For the kinetic studies, normalised reflectance values at $560 \mathrm{~nm}$ were generated according to the formula:

$$
\mathrm{N}=\left(\mathrm{R}-\mathrm{R}_{\mathrm{MC}}\right) /\left(\mathrm{R}_{\mathrm{SP}}-\mathrm{R}_{\mathrm{MC}}\right)
$$

10 where $\mathrm{N}$ is the normalised reflectance value at time $\mathrm{t}, \mathrm{R}$ is the reflectance value at time $t$ and $R_{M C}$ and $R_{S P}$ are the initial reflectance values of the SP and $\mathrm{MC}$ forms, respectively.

\section{Comparison of LEDs for SP $\leftrightarrow$ MC switching}

15 The thin layer of sedimented microbeads with covalently immobilised SP using an eight-carbon spacer $(0.01 \mathrm{~g}$ in $1.3 \mathrm{ml}$ of ethanol) was exposed to different LEDs in order to evaluate their capabilities for switching the surface immobilised spiropyran in a series of parallel experiments.

20 First $\mathrm{SP} \rightarrow \mathrm{MC}$ switching was induced by one minute exposure of the bead layer to a $375 \mathrm{~nm}$ LED.

Following this, the MC form was irradiated for 1 minute with white $(430-760 \mathrm{~nm})$, blue $(430 \mathrm{~nm})$, green $(525 \mathrm{~nm})$, and red $(630$ $\mathrm{nm}$ ) LEDs in order to compare the effectiveness of each LED to

25 induce $\mathrm{MC} \rightarrow \mathrm{SP}$ switching. After each irradiation the sample vials were placed in the holder to measure the reflectance spectrum.

\section{Evaluation of the spiropyran immobilisation strategies}

30 The influence of immobilisation strategy on switching efficiency was evaluated by repeatedly cycling three different spiropyran functionalised microbead samples $(0.01 \mathrm{~g}$ in $1.3 \mathrm{ml}$ of ethanol) between the SP and MC forms using UV and white LEDs according to the following procedure:

35 1. The microbead layer was irradiated for $1 \mathrm{~min}$ with a white LED.

2. The reflectance spectrum was immediately captured by placing the vial into the specially constructed holder.

3. The microbead layer was then irradiated with a UV LED for $1 \mathrm{~min}$.

4. The reflectance spectrum was again captured using the same procedure as in (2), above.

Each cycle was repeated ten times for the three different 45 spiropyran immobilisation strategies:

(a) Physical adsorption (no covalent binding) - SP(PA);

(b) Covalent immobilisation using an four-carbon spacer between the spiropyran and the surface of the beads $\mathrm{SP}(\mathrm{C}-4)$

50 (c) Covalent immobilisation using an eight carbon spacer between the spiropyran and the surface of the beads $\mathrm{SP}(\mathrm{C}-8)$.

\section{Influence on solvents on $\mathrm{MC} \rightarrow \mathrm{SP}$ kinetics}

${ }_{55}$ The kinetics of ring closing (i.e. $\mathrm{MC} \rightarrow \mathrm{SP}$ conversion) of the $\mathrm{SP}(\mathrm{C}-8)$ sample was investigated in four different solvents: water, ethanol, methanol and acetonitrile. Other solvents, such as acetone, ethyl acetate, hexane and chloroform were found to destroy the polymeric matrix. In each kinetic evaluation 60 experiment the bead sample was washed 6 times with the corresponding solvent. Each washing stage included:

1. Centrifugation of $1.3 \mathrm{ml}$ suspension of $0.01 \mathrm{~g}$ of functionalized microbeads for $3 \mathrm{~min}$ at $4000 \mathrm{rpm}$.

2. Removal of the supernatant solvent and addition of 1.3 $\mathrm{ml}$ of fresh solvent
3. Sonication of the suspension for $5 \mathrm{~min}$.

4. Further centrifugation.

The resulting thin sedimented layer was subsequently exposed for 701 minute to a white LED followed by irradiation with a $375 \mathrm{~nm}$ UV LED for 1 minute. Then the sample was placed in the vial holder and reflectance measurements taken at $0,1,5,15,30,60$, $100,140,180,220,260,300$ minutes. During the experiment, the vial was kept in the dark inside the holder and measurements 75 were taken manually at the above fixed time intervals, turning the halogen light on just for the few seconds needed to capture the spectrum, in order to minimise re-conversion to the SP form by the spectrometer white light source.

\section{Photostability evaluation of the surface immobilised spiropyran}

The photostability of the surface immobilised spiropyran was evaluated by repeatedly cycling two $-\mathrm{SP}(\mathrm{C}-8)$ freshly prepared samples $(0.01 \mathrm{~g}$ in $1.3 \mathrm{ml}$ of ethanol) between the SP and $\mathrm{MC}$

85 forms using UV and white LEDs. Cycles were performed according to the procedure reported for the evaluation of the spiropyran immobilisation strategies. Each cycle was repeated 50 times monitoring the reflectance values at $560 \mathrm{~nm}$.

\section{Evaluation of $\mathrm{Ca}^{2+}$ and $\mathrm{Cu}^{2+}$ interactions with SP-coated microbeads}

Spiropyran coated microbeads (-SP(C-8) sample) suspended in ethanol and converted to the $\mathrm{MC}$ form were exposed to the same concentrations of $\mathrm{Ca}^{2+}$ and $\mathrm{Cu}^{2+}$ ethanolic solutions in order to 95 evaluate the complex formation at the bead surface.

Each experiment was carried out using the following procedure:

1. Centrifugation of a suspension of $0.01 \mathrm{~g}$ of functionalised microbeads in $1.3 \mathrm{ml}$ of ethanol for 3 minutes at 4000 rpm.

2. Exposure of the resulting thin bead layer to a white LED for 1 minute (promotes MC conversion to the SP form)

3. Recording of the reflectance spectrum of the colourless SP form.

4. Exposure of the thin layer to a UV LED for 1 minute (promotes SP conversion to the MC form).

5. Recording of the reflectance spectrum of the coloured $\mathrm{MC}$ form.

6. Addition of $0.1 \mathrm{ml}$ of $10^{-2} \mathrm{M} \mathrm{Ca}^{2+}$ or $\mathrm{Cu}^{2+}$ ethanolic solutions (final concentration $7.1 \times 10^{-4} \mathrm{M}$ ) followed by sonication for 5 minutes

7. Centrifugation of the microbeads for $3 \mathrm{~min}$ at $4000 \mathrm{rpm}$

8. Recording of the reflectance spectrum.

9. Exposure of the thin bead layer to a white LED for 1 minute (promotes metal expulsion and $\mathrm{MC}$ conversion to the SP form)

10. Copious washing ( $\mathrm{x} 6$ times) with fresh ethanol

\section{Results and Discussion}

\section{Solution studies}

120 In ethanol solution upon irradiation of the colourless SP form with UV light, conversion to the MC form occurred, which was accompanied by the appearance of the characteristic strong absorbance band in the UV-VIS spectrum around $540 \mathrm{~nm}$.

When placed in contact with increasing concentrations of certain 125 metals, particularly transition metals ${ }^{5}, 7$, this $540 \mathrm{~nm}$ band decreases, accompanied by an increase in absorbance towards the blue end of the spectrum. 

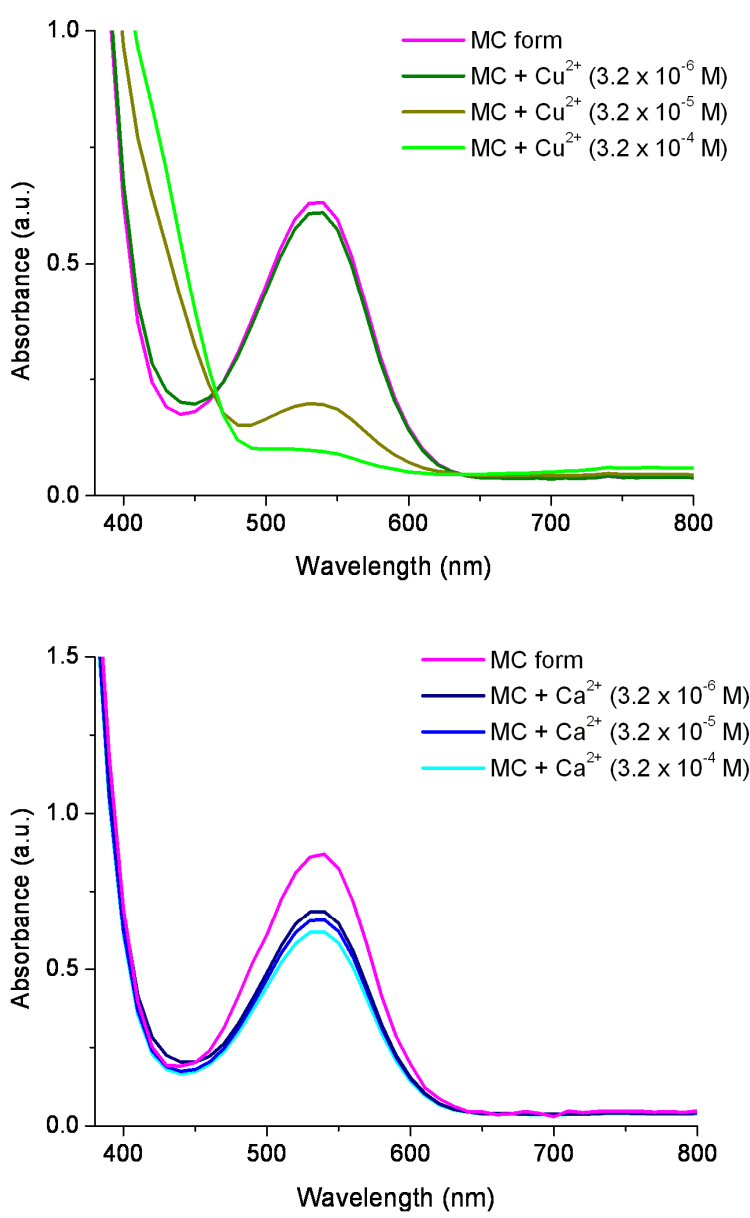

5 Figure 4: Absorbance changes in the spectra of the $\mathrm{MC}$ form $\left(10^{-3} \mathrm{M}\right.$ in ethanol) when placed in contact with $\mathrm{Cu}^{2+}$ and $\mathrm{Ca}^{2+}$ ions at different molar concentrations in ethanol solution.

10 The interaction between the phenolate group of the MC form and these metal ions is not particularly selective, but according to our solution studies, $\mathrm{Cu}^{2+}$ ions in ethanol cause the most appreciable colour change. In the presence of $\mathrm{Cu}^{2+}$, the $540 \mathrm{~nm}$ absorbance band decreases, and the absorbance at $430 \mathrm{~nm}$ increases, resulting 15 in a clear colour change from purple to orange-yellow (Fig. 4).

These effects are in accordance with literature reports of UV-vis absorbance changes accompanying $\mathrm{MC}-\mathrm{Cu}^{2+}$ complexation ${ }^{5-7,10}$. It is generally agreed that these effects are caused by the formation of a 2:1 mole ratio complex, with two MC molecules 20 bound to each $\mathrm{Cu}^{2+}$ ion $^{10}$. Solution studies show that corresponding additions of $\mathrm{Ca}^{2+}$ ions to the $\mathrm{MC}$ solution does not cause any appreciable visual colour or spectral change.

In contrast to the dramatic spectral and colour changes occurring in the presence of $\mathrm{Cu}^{2+}$, in the case of $\mathrm{Ca}^{2+}$ ions, the only effect 25 observed is a slight decrease in the absorbance of the MC form at $\lambda_{\max }(540 \mathrm{~nm})$ which is indicative of a general change in the local environment (such as increasing ionic strength) rather than a specific interaction.

However, it is suggested that a relatively strong $\mathrm{MC}-\mathrm{Ca}^{2+}$ 30 interaction occurs when two $\mathrm{MC}$ units belonging to benzospiropyran amide derivative are linked by an acetamidopropane linker to form a bis-benzospiropyranindoline bidentate ligand ${ }^{26}$. The binding is eight times higher than that achieved with a single spiropyran amide derivative obtained from 35 the separation of the two units.

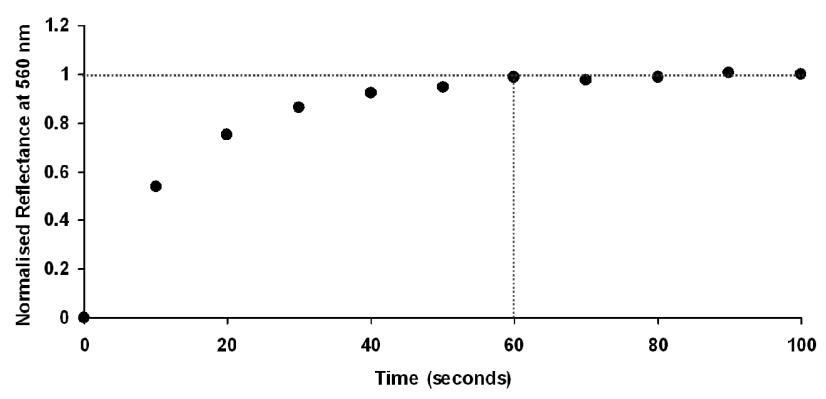

Figure 5: Normalised reflectance values recorded during UV LED irradiation $(375 \mathrm{~nm})$ of an $-\mathrm{SP}(\mathrm{C} 8)$ microbead sample $(0.01 \mathrm{~g}$ in $1.3 \mathrm{ml}$ of 40 ethanol) showing that the maximum reflectance value for the conversion to the $\mathrm{MC}$ form (plateau) is reached in $60 \mathrm{sec}$.

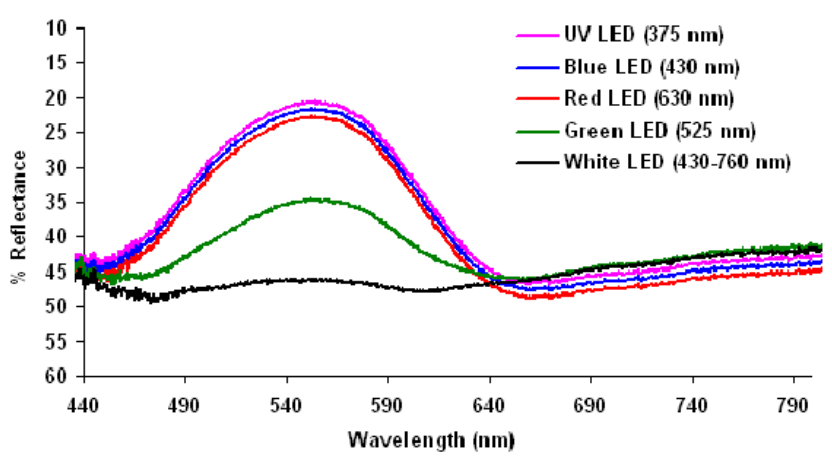

45 Figure 6: Reflectance spectra of an -SP(C-8) microbead sample (0.01g in $1.3 \mathrm{ml}$ of ethanol) after one minute irradiation with UV LED (to promote the conversion to the MC form) and subsequent exposure to blue, red, green and white LED to promote the reconversion to the SP form.

50

This result again demonstrates the importance of preorganisation of the ligand to form sandwich-type bidentate complexes with doubly charged cations. Other examples of enhanced metal binding have been achieved by attaching crown ether moieties 27

55 to the spiropyran unit. However, in the case of the unmodified benzospiropyran unit used in these studies (1), there is no evidence of any significant $\mathrm{MC}-\mathrm{Ca}^{2+}$ formation.

In view of these differences, it was decided to focus on $\mathrm{Cu}^{2+}$ and $\mathrm{Ca}^{2+}$ ions for the preliminary evaluation of the modified beads ${ }_{60}$ binding behaviour, as examples of binding $\left(\mathrm{Cu}^{2+}\right)$ and nonbinding guests $\left(\mathrm{Ca}^{2+}\right)$.

\section{Optical properties of spiropyran coated microbeads}

The covalently bound spiropyran on the microbeads can be effectively switched between the MC (pink) and SP (white) ${ }_{65}$ forms. Upon irradiation with a UV-LED $(375 \mathrm{~nm})$ the beads become purple in colour, and the characteristic large absorption band around $560 \mathrm{~nm}$ appears.

We have previously demonstrated that the use of LEDs instead of more powerful light sources substantially reduces the well known 70 photobleaching process that occurs when the switching equilibrium between the SP and the MC form is performed extensively over time ${ }^{28}$. For example, the LEDs we are using are typically ca. $1 \mathrm{~mW} / \mathrm{cm}^{2}$ compared to the power of arc lamp light sources reported in most papers, which are typically 50-100 W. 


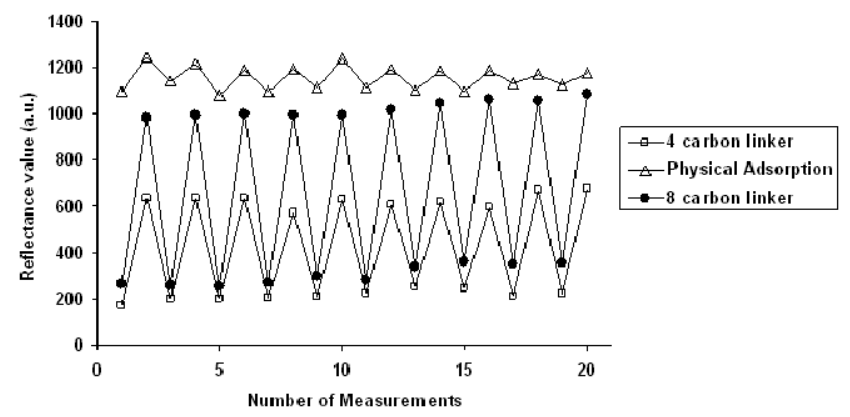

Figure 7: Reflectance intensity at $560 \mathrm{~nm}$ of the bead surface functionalised with different immobilisation strategies after repeated switching cycles consisting of 1 minute UV-LED irradiation followed by 51 minute white-LED irradiation.

Despite this large reduction in emission power, the switching efficiency is relatively unaffected and repeated switching can be 10 successfully performed.

We have also investigated how the LEDs emission wavelength affects their ability to switch the immobilised spiropyran.

UV LEDs at $375 \mathrm{~nm}$ exhibit fast and efficient $\mathrm{SP} \rightarrow \mathrm{MC}$ switching, with the initial response occurring within a few 15 seconds, reaching full conversion to the MC form in $60 \mathrm{~s}$ (Fig. 5). Green $(525 \mathrm{~nm})$, red $(638 \mathrm{~nm})$, blue $(426 \mathrm{~nm})$ and white LEDs have also been tested for MC-SP switching.

It was found that after 1 minute irradiation of the $\mathrm{MC}$ form with red or blue LEDs, the corresponding reflectance spectra exhibit a 20 consistent $560 \mathrm{~nm}$ band, indicating that the coloured $\mathrm{MC}$ is still the predominant form. However after 1 minute exposure to green and white LEDs the MC peak decreases significantly, indicating a much more efficient conversion to the SP form (Fig. 6).

Comparing the green and white LEDs, the white is clearly the 25 more efficient: after 60 seconds exposure of the purple $\mathrm{MC}$ form on the bead surface to the white LED, the SP form is fully regenerated, while after 1 minute irradiation with the green LED a small band around $560 \mathrm{~nm}$ is still present, although much reduced compared to equivalent exposures using the red and the 30 blue LEDs. It was previously demonstrated ${ }^{4}$ that the green region is the more active region of the visible spectrum for the backconversion of MC to the SP form.

From these results, it can be concluded that effective $\mathrm{SP} \rightarrow \mathrm{MC}$ switching on the microbeads can be achieved through 1 minute 35 irradiation with a UV LED, while the reverse $\mathrm{MC} \rightarrow$ SP switching can be achieved through 1 minute irradiation with a white LED. The switching efficiency of the surface immobilised spiropyran is also strongly influenced by the presence of a spacer group between the active SP unit and the bead surface. When the 40 spiropyran derivative is simply physically adsorbed on the surface of the beads (after 72 hours of continuous stirring), UV LED irradiation generates almost no colour change. After 1 minute exposure, a faint pink colour appears on the beads and in the supernatant, indicating that $\mathrm{SP} \rightarrow \mathrm{MC}$ switching is not 45 effective, and that there is a degree of leaching of SP from the beads, even after repeated washing. Ethanol suspensions of two types of covalently functionalised microbeads were also examined one with a four carbon linker and another with an eight carbon linker between the spiropyran and the polymeric surface.

50 These, and beads with physically adsorbed spiropyran were repeatedly exposed ( $\mathrm{x} 20$ times) alternatively to 1 minute exposure with a UV-LED followed by 1 minute with a whiteLED (Fig. 7).

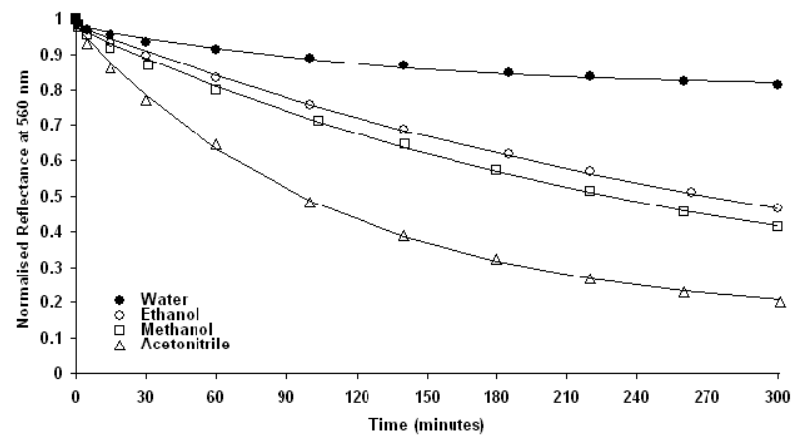

F5 55 igure 8: Normalised reflectance values at $560 \mathrm{~nm}$ of the covalently immobilised SP (8-carbon linker) on the bead surface in different solvents. The decrease in reflectance intensity indicates $\mathrm{MC}$ form reverting to SP form in the dark after irradiation with a UV-LED. The

60 residual error calculated between the data and the model in between does not exceed $3 \%$ at any point in the fitted curve.

\begin{tabular}{lc}
\hline \multicolumn{1}{c}{ Solvent } & $\mathrm{k}_{\mathrm{R}}\left(\mathbf{m i n}^{-1}\right)$ \\
\hline Water & $7.9( \pm 0.1) \times 10^{-3}$ \\
\hline Methanol & $3.8( \pm 0.1) \times 10^{-3}$ \\
\hline Ethanol & $2.9( \pm 0.1) \times 10^{-3}$ \\
\hline Acetonitrile & $9.1( \pm 0.2) \times 10^{-3}$ \\
\hline
\end{tabular}

Table 1: Rate constants for the reversion of MC to SP obtained from the 65 best-fit curves in figure 8 .

The percentage reflectance values at $560 \mathrm{~nm}$ were recorded and the plot clearly shows a high efficiency switching of the beads 70 with the covalently bonded spiropyran while almost no conversion to the MC form is observed on the beads where spiropyran is physically adsorbed.

In contrast, the covalently bound spiropyran appears to be free enough from the surface to be well solvated by the ethanol, which 75 allows the degree of conformational flexibility required for efficient switching between the MC and SP forms. It was previously demonstrated that the use of tethers between a polymeric surface and spiropyran units can dramatically improve the switching efficiency and binding ability, as the molecular re80 arrangement that accompanies $\mathrm{SP} \leftrightarrow \mathrm{MC}$ photoswitching is inhibited if the spiropyran molecule is too close to the surface ${ }^{4}$. It seems therefore, that when the spiropyran is physically adsorbed into the microbead surface it is too conformationally restricted for effective switching. Furthermore, considering the 85 length of the covalent tether between the bead surface and the spiropyran unit, the eight carbon spacer was almost twice as efficient at switching compared to the four carbon linker.

In view of these results, the eight carbon tether was used in all subsequent experiments to compare the ring closing relaxation 90 kinetics in several solvents.

This involved inducing the formation of the MC state by exposure to the UV-LED for 1 minute, and recording reflectance spectra at fixed time intervals as equilibrium is re-established between the SP and MC forms. It is known from solution studies 95 that this process follows first order kinetics ${ }^{29}$, with the thermodynamics and kinetics strongly influenced by the solvent. The use of SP-functionalised microbeads enables the switching behaviour to be examined in a wider range of solvents, including water, in which spiropyran is not soluble. During these 100 measurements, the sample is kept in the dark to avoid the influence of the ambient light on the reconversion to the SP form (Fig. 8). 
The first order rate constants were estimated by fitting the normalised reflectance values at $560 \mathrm{~nm}$ using Microsoft Excel Solver ${ }^{30,28}$ using the following equation (Table 1):

$$
5=a e^{-k t}+b
$$

where $\mathrm{y}$ is the normalised reflectance at $560 \mathrm{~nm}$, a is the normalised reflectance at time $0, \mathrm{k}$ is the rate constant and $\mathrm{b}$ is the asymptotic value.

${ }_{10}$ Our results show that the equilibrium between the two forms strongly depends on the solvent type, which suggests that the surface immobilised dye is actively interacting with the solvent molecules. For example, in water the conversion of the $\mathrm{MC}$ to the SP form is strongly inhibited, and after 300 minutes MC is still 15 the predominant form. This behaviour is not surprising, as the zwitterionic $\mathrm{MC}$ form is stabilised in highly polar environments compared to the more hydrophobic SP form.

Methanol and ethanol exhibit intermediate behaviour between water and acetonitrile. For example, after 5 hours the $\mathrm{MC}$ form is 20 still present at around $50 \%$ of its initial concentration. In acetonitrile after 300 minutes, the MC has been almost fully converted to the SP form. Considering the kinetic values, methanol $\left(3.8 \pm 0.1 \times 10^{-3} \mathrm{~min}^{-1}\right)$ and ethanol $\left(2.9 \pm 0.1 \times 10^{-3}\right.$ $\left.\mathrm{min}^{-1}\right)$ are pretty close, even though, the rate constant obtained in

25 methanol is slightly larger. Interestingly the value obtained for water $\left(7.9 \pm 0.1 \times 10^{-3} \mathrm{~min}^{-1}\right)$ is similar to that of acetonitrile $(9.1$ $\left.\pm 0.2 \times 10^{-3} \mathrm{~min}^{-1}\right)$, despite the fact that the steady-state greatly favours MC in water, and SP in acetonitrile.

In general, the obtained values are high when compared to 30 relaxation times obtained for spiropyran doped polystyrene films ${ }^{28}$, where the rate of the spiropyran closing is around $10^{-2}$ $\sec ^{-1}$. However, in those studies the SP was entrapped within a polymer layer, whereas in this case, the SP is primarily surface immobilised on a microbeads shaped suspended polymer.

35

Another issue to be considered when dealing with spiropyran photochromic dyes is their photostability over time, due to a well documented photobleaching process ${ }^{3,31-34}$ that occurs when the molecule is exposed to UV-vis radiation for extended time 40 periods. It is generally agreed that photodegradation is enhanced by the presence of free radical or singlet oxygen, by the formation of MC aggregates and by the light source used (especially in the UV region). It has been demonstrated that the introduction of antioxidant groups as free radical scavengers or the reduction of ${ }_{45} \mathrm{MC}$ aggregation remarkably reduces the photodegradation ${ }^{28,33}$. In the same way, moving to low power light sources such as LEDs (less photon density) and the immobilisation of the spiropyran on the bead surface should reduce the extent of photodegradation, as was demonstrated previously with SP covalently immobilised on 50 a polymethylmetacrylate film ${ }^{10}$. In this work, the switching efficiency in ethanol was evaluated by normalising the difference in the reflectance value at $560 \mathrm{~nm}$ to the initial values:

$$
\% \mathrm{E}=\left[\left(\mathrm{R}_{\mathrm{SPi}}-\mathrm{R}_{\mathrm{MCi}}\right) /\left(\mathrm{R}_{\mathrm{SP}}-\mathrm{R}_{\mathrm{MC}}\right)\right] \times 100
$$

where $\mathrm{E}$ is the percent efficiency, $\mathrm{R}_{\mathrm{SPi}}$ and $\mathrm{R}_{\mathrm{MCi}}$ are the initial values of reflectance of the $\mathrm{MC}$ and the SP forms when the initial switching was performed and $R_{S P}$ and $R_{M C}$ are the reflectance values obtained in subsequent switching cycles.

60 According to this model, $100 \%$ is the maximum efficiency, when no photodegradation occurs, while $0 \%$ is when the photochromic dye is completely photobleached. (a)

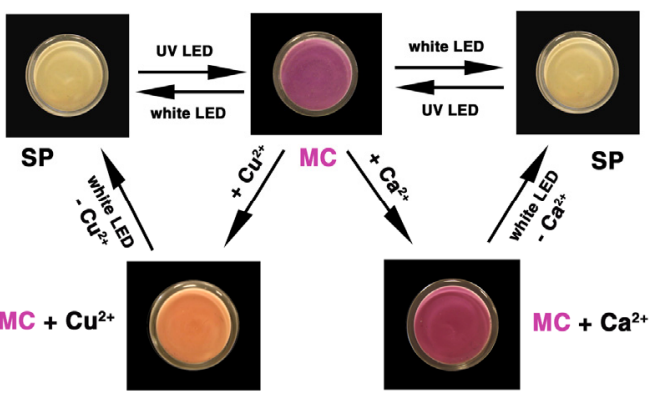

70 (b)

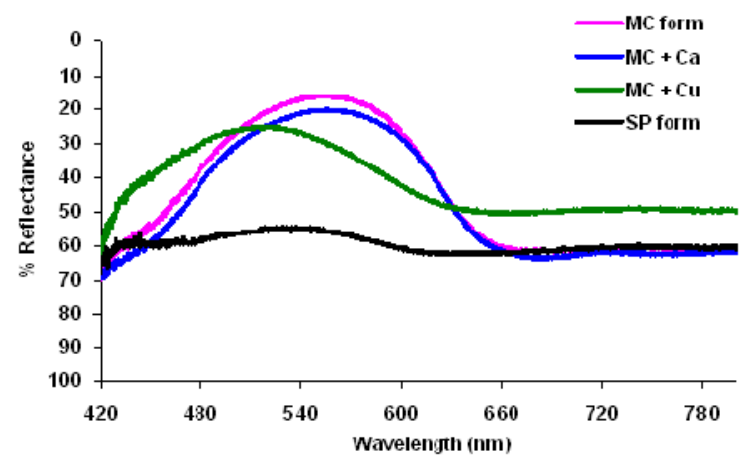

Figure 9: (a) Microbead colour changes at the bottom of the glass vial ($\mathrm{SP}(\mathrm{C}-8)$ sample, $0.01 \mathrm{~g}$ in $1.3 \mathrm{ml}$ of ethanol) observed during switching 75 between the SP and MC forms, and in the presence of $\mathrm{Cu}^{2+}$ and $\mathrm{Ca}^{2+}$ ions (final concentration $7.1 \times 10^{-4} \mathrm{M}$ in ethanol). (b) Reflectance spectra of the colour changes: in the presence of $\mathrm{Cu}^{2+}$ the $560 \mathrm{~nm}$ peak shift around $520 \mathrm{~nm}$ with an increase around $445 \mathrm{~nm}$, in the presence of $\mathrm{Ca}^{2+}$ no detectable spectra change is recorded.

80

After 50 switching cycles (one minute irradiation with UV LED followed by one minute exposure to white LED), the efficiency was found to decrease by around $40 \%$ with respect to the initial 85 switching values in two samples of freshly prepared functionalised $-\mathrm{SP}(\mathrm{C} 8)$ microbeads. For both samples the final percent efficiency value is around $60 \%$ which directly correspond to the ratio of surviving MC.

This is an improvement compared to the solution studies reported 90 by $\mathrm{Li}$ et al. in which the percentage of surviving MC (1) in methanol after just 13 switching cycles (with a 30 second UV irradiation for each cycle, using high pressure mercury lamp) is around $56.4 \%{ }^{3}$. However, it is not as efficient as other polymeric immobilisation strategies using a film matrix, where after more 95 than 370 cycles using LEDs the decrease in photostability is around $14 \%(86 \% \text { of surviving MC })^{10}$. This is probably due to the fact that the microbeads are free to move and interact between each other, allowing eventual MC aggregation.

\section{Ion-binding behaviour of spiropyran coated microbeads}

Spiropyran functionalised microbeads offer the interesting possibility of controlling metal ion uptake using light, as only the MC form binds with metal ions. It should, therefore, be possible to determine when and where ion binding occurs (e.g. by 105 illuminating with a UV-LED), and as the beads are mobile, the bound ions can be transported to another location, at which the ions can be released by illumination with a white LED. 


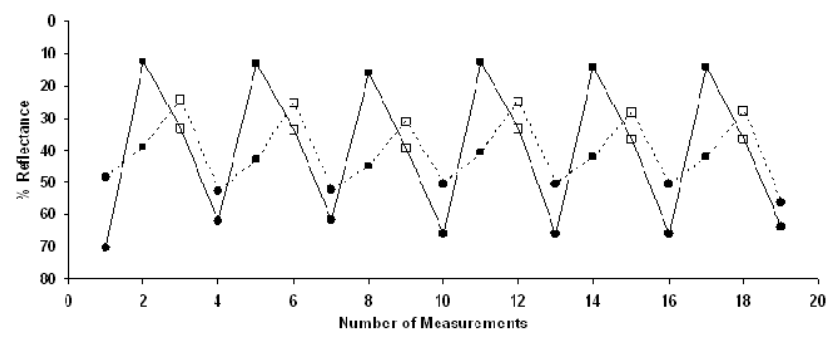

Figure 10: Switching cycles of a $-\mathrm{SP}(\mathrm{C}-8)$ microbead sample $(0.01 \mathrm{~g}$ in $1.3 \mathrm{ml}$ of ethanol) between the SP (full circle), $\mathrm{MC}$ (full square) form and

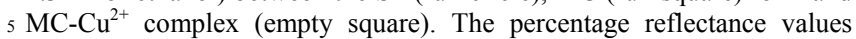
have been evaluated at $560 \mathrm{~nm}$ (continuous line) and at $445 \mathrm{~nm}$ (dotted line).

${ }_{10}$ Once the beads are reconverted again to the $\mathrm{MC}$ form, the surface is ready for re-use. Furthermore, the system is inherently selfindicating, and by monitoring the colour, it can be seen which form is present (SP or MC) and whether ions are bound (colour/UV-vis spectrum changes again).

${ }_{15}$ Based on previous solution studies, $\mathrm{Cu}^{2+}$ ions were chosen as an example of self-indicating complex formation, while $\mathrm{Ca}^{2+}$ ions were chosen as an example of non-complex formation. When the purple MC-activated beads are placed in contact with a copper (II) nitrate solution they undergo a further colour change from ${ }_{20}$ pink to orange. The change is reversible, upon irradiation with white light and replacement of the supernatant with fresh solvent, the SP form is restored, the metal is expelled, and the beads can be converted again to the MC form ready for another ion binding cycle. When the same experiment is repeated using instead $\mathrm{Ca}^{2+}$ 25 ions at the same concentration, in the same solvent and with the same counter ion, no colour change is detected (Fig. 9).

These colour changes can be monitored by recording the UV-vis reflectance spectra. In the presence of $\mathrm{Cu}^{2+}$ ions the $560 \mathrm{~nm}$ band decreases and is shifted towards a lower wavelength (around 520 $30 \mathrm{~nm}$ ) and another band appears around $445 \mathrm{~nm}$ (Fig. 9). This process is fully reversible, as shown in (Fig. 10), which shows 6 repeat cycles through $\mathrm{SP}, \mathrm{MC}, \mathrm{MC}-\mathrm{Cu}^{2+}$ complex, and back to SP. The spectra show that at $560 \mathrm{~nm}$ the MC has the highest absorbance while the $\mathrm{MC}-\mathrm{Cu}^{2+}$ complex has much lower value 35 (the $560 \mathrm{~nm}$ band decreases in the presence of the metal ion), while the SP form has the lowest of all, as expected as this form has no absorbance in this region of the UV-vis spectrum. In contrast, at $445 \mathrm{~nm}$, the $\mathrm{MC}-\mathrm{Cu}^{2+}$ has the highest absorbance whereas the $\mathrm{MC}$ and SP form values are lower.

40 In comparison, the presence of $\mathrm{Ca}^{2+}$ causes no change in the 560 nm MC band (Fig. 9), and therefore the spiropyran microbeads system can definitely differentiate between $\mathrm{Ca}^{2+}$ and $\mathrm{Cu}^{2+}$ ions.

We have found that $\mathrm{Cu}^{2+}$ produces the most striking blue-shift upon binding with $\mathrm{MC}$, though other ions such as $\mathrm{Co}^{2+}$ do cause a 45 smaller shift in the MC spectra upon binding ${ }^{4}$.

\section{Conclusions}

We can conclude that the functionalised microbeads exhibit ionbinding behaviour and they can be photo-reversibly switched between an active (binding) and an inactive (non-binding) form. ${ }_{50}$ The active form selectively retains $\mathrm{Cu}^{2+}$ but not $\mathrm{Ca}^{2+}$ whereas the inactive form (SP) does not retain either ion. These results show that spiropyran modified beads can be used for the photocontrolled selective accumulation and release of ions. Furthermore, the system is inherently self-indicating, as each
${ }_{55}$ form (SP, MC, MC-ion complex) has a different colour and UVvis absorbance spectrum.

On the basis of this behaviour, the foundation for a photocontrolled system for retention and elution of metal ions can be laid: irradiation of the stationary phase with UV-LEDs causes 60 retention of guest species due to the presence of the MC form, while subsequent exposure to white LEDs causes release of guest species into the mobile phase, providing a potential route to photodynamic liquid chromatography. Beads can be easily packed into capillary column that could be used for photo${ }_{65}$ controlled separations. This concept could also be usefully applied generally to photo-accumulation of ions, their transport to other locations and subsequent photo-release. This could, for example, facilitate localised control of the chemical environment at specific locations within a microfluidic or bio-circulation 70 system.

\section{Acknowledgements}

The authors would like to acknowledge support for this research by Science Foundation Ireland under the 'Adaptive Information ${ }_{75}$ Cluster' award (SFI 03/IN.3/1361 and 07/RPF/MASF812) and EC FP6 Marie Curie Excellence Grants fellowship.

\section{Notes and references}

a Adaptive Sensors Group, National Centre for Sensor Research, ${ }_{80}$ Glasnevin, Dublin 9, Ireland. Tel:00353(0)17005404 E-mail: dermot.diamond@dcu.ie

${ }^{b}$ School of Chemical Sciences and National Centre for Sensor Research, Glasnevin, Dublin 9, Ireland Tel: 00353(0)17005611 and 00353(0)17005060 E-mail: mirek.macka@dcu.ie and brett.paull@dcu.ie

85

1. V. I. Minkin, Chemical Reviews, 2004, 104, 2751-2776.

2. H. Dürr and L. H. Bouas, Photochromism - molecules and systems, Elsevier, 2003.

3. X. Li, J. Li, Y. Wang, T. Matsuura and J. Meng, Journal of

90 Photochemistry and Photobiology A: Chemistry, 2004, 161, 201-213.

4. R. J. Byrne, S. E. Stitzel and D. Diamond, Journal of Materials Chemistry, 2006, 16, 1332-1337.

5. H. Görner and A. K. Chibisov, Journal of the Chemical Society, Faraday Transactions, 1998, 94, 2557-2564.

95 6. J.-W. Zhou, Y.-T. Li and X.-Q. Song, Journal of Photochemistry and Photobiology A: Chemistry, 1995, 87, 37-42.

7. A. K. Chibisov and H. Gorner, Chemical Physics, 1998, 237, 425442.

8. T. Suzuki, T. Kato and H. Shinozaki, Chemical Communication, 2004, 2036-2037.

9. N. Shao, Y. Zhang, S. Cheung, R. Yang, W. Chan, T. Mo, K. Li and F. Liu, Analytical Chemistry, 2005, 77, 7294-7303.

10. A. Radu, S. Scarmagnani, R. Byrne, C. Slater, K. T. Lau and D. Diamond, Journal of Physics D: Applied Physics, 2007, 7238-7244.

105 11. M.-S. Kim, W.-J. Chung, S.-H. Cho, B.-G. Kim, Y.-S. Lee and Y.-K. Kim, Transducers, 2003.

12. X. Guo, D. Zhang, G. Zhang, Y. Guan and D. Zhu, Chemical Physics Letters, 2004, 398, 93-97.

13. T. Fujiwara, J. Harada and K. Ogawa, Journal of Physical Chemistry B, 2004, 108, 4035-4038.

14. R. P. Paradkar and R. R. Williams, Applied Spectroscopy, 1996, 50, 753-758. 
15. E. K. Paleologos, M. I. Prodromidis, D. L. Giokas, A. C. Pappas and M. I. Karayannis, Analytica Chimica Acta, 2002, 467, 205-215.

16. S. Koji and N. Toshihiko, Journal of Photopolymer Science and Technology, 2001, 14, 233-238.

5 17. V. K. Hughes, P. S. Ellis, T. Burt and N. E. I. Langlois, Journal of Clinical Pathology, 2004, 57, 355-359.

18. J. Gade, D. Palmqvist, P. Plomgård and G. Greisen, 2006, 51, 121136.

19. A. Balkenius and A. Kelber, Journal of Experimental Biology, 2004, 207, 3307-3316.

20. C. M. Hofmann, T. W. Cronin and K. E. Omland, Evolution, 2006, 60, 1680-1691.

21. S. Yoshioka and S. Kinoshita, Journal of the Optical Society of America A, 2006, 23, 134-141.

15 22. R. Rosario, D. Gust, M. Hayes, F. Jahnke, J. Springer and A. A. Garcia, Langmuir, 2002, 18, 8062-8069.

23. D. C. MacLaren and M. A. White, Journal of Materials Chemistry, 2003, 13, 1701-1704.

24. A. Pauw, American Journal of Botany, 2006, 93, 917-926.

20 25. L. M. Mäthger and R. T. Hanlon, Biology Letters, 2006, 2, 494-496.

26. J. Filley, M. A. Ibrahim, M. R. Nimlos, A. S. Watt and D. M. Blake, Journal of Photochemistry and Photobiology A: Chemistry, 1998, 117, 193-198.

27. M. V. Alfimov, O. A. Fedorova and S. P. Gromov, Journal of

25 Photochemistry and Photobiology A: Chemistry, 2003, 158, 183-198.

28. S. Stitzel, R. Byrne and D. Diamond, Journal of Materials Science, 75 2006, 41, 5841-5844.

29. H. Gorner, Physical Chemistry Chemical Physics, 2001, 3, 416-423.

30. D. Diamond and V. C. A. Hanratty, Spreadsheet Application in

$30 \quad$ Chemistry using Microsoft Excel, Wiley, New York, 1997.

31. G. Baillet, G. Giusti and R. Guglielmetti, Journal of Photochemistry and Photobiology A: Chemistry, 1993, 70, 157-161.

32. G. Baillet, M. Campredon, R. Guglielmetti, G. Giusti and C. Aubert, Journal of Photochemistry and Photobiology A: Chemistry, 1994, 83,

$35 \quad 147-151$

33. R. Matsushima, M. Nishiyama and M. Doi, Journal of 85 Photochemistry and Photobiology A: Chemistry, 2001, 139, 63-69.

34. R. Demadrille, A. Rabourdin, M. Campredon and G. Giusti, Journal of Photochemistry and Photobiology A: Chemistry, 2004, 168, 143152. 